\title{
Enzyme-linked immunosorbent assay (ELISA) using antibody class capture for the detection of antitoxoplasma IgM
}

\author{
RONALD A PAYNE, MICHAEL ISAAC, JANET M FRANCIS
}

From The Public Health Laboratory, Cockett Road, Swansea, S Wales

SUMMARY Sera from 180 patients with suspected toxoplasmic lymphadenopathy were examined for antitoxoplasma IgM by an enzyme-linked immunosorbent assay (ELISA), using antibody class capture (ACCA). Of 82 positive ACCA results, 78 were confirmed by testing the IgM fractions of the sera, obtained by sucrose density gradient centrifugation (SDGC). The four positive results which could not be confirmed were all from patients with at least a year's history of lymphadenopathy. Sera from 10 patients with low Sabin Feldman dye test (DT) titres gave positive ACCA results and subsequent specimens from them showed a rise in antibody concentration, confirming the diagnosis of acute toxoplasmosis.

The antitoxoplasma IgM immunofluorescent antibody test (IgM-IFA) on whole serum was relatively insensitive and gave false-positive results with sera containing rheumatoid factor (RF) and antinuclear factor (ANF). There were no false-positive ACCA results with such sera, probably because the conjugates were prepared from $F\left(a b^{\prime}\right)_{2}$ fragments of antitoxoplasma serum.

The ACCA proved to be sensitive, specific and easily automated enabling examination of large numbers of specimens.

The diagnostic value of detecting specific immuno globulin $\mathbf{M}$ (IgM) in acute and congenital toxoplasmosis is well established. ${ }^{1}$ The antitoxoplasma IgM immunofluorescent antibody test (IgM-IFA) is widely used for this purpose but has been shown to have serious disadvantages. False-positive results often occur when either rheumatoid factor (RF) or antinuclear factor (ANF) are present in the patient's serum..$^{23}$ False-negative results may occur when high concentrations of antitoxoplasma IgG are present because they compete for antigenic sites and inhibit the demonstration of antitoxoplasma IgM. ${ }^{4}$ Other methods employ the physical separation of IgM from IgG by either sucrose density gradient centrifugation (SDGC) or exclusion chromatographys followed by serological tests in the presence and absence of 2-mercaptoethanol (2-ME) as has been described for the detection of antirubella $\operatorname{IgM} .^{6}$ These fractionation techniques are time-consuming, require expensive apparatus and are not practicable for large numbers of specimens.

An enzyme-linked immunosorbent assay

Accepted for publication 5 Janusary 1982
(ELISA) using antibody class capture assay for the detection of antitoxoplasma IgM (ACCA) is an alternative to the above methods. IgM can be separated from other immunoglobulins by selective binding to antihuman $\mu$ chain immunoglobulin coated onto plastic material. The antitoxoplasma activity of captured IgM can then be assayed by adding toxoplasma antigen followed by enzyme-labelled antibody as described for hepatitis $A$ antigen. ${ }^{7}$ The development and use of an ACCA in cases of toxoplasma lymphadenopathy is described. Its possible use in testing large numbers of specimens is discussed.

\section{Material and methods}

SPECIMENS

Serum samples were obtained from 180 patients who had lymphadenopathy, with dye test ${ }^{8}$ (DT) titres ranging from $<1 / 4$ to $1 / 4096$ or greater $(<2$ $2000 \mathrm{IU} / \mathrm{ml}$ ), 20 patients whose sera contained RF, three patients whose sera contained ANF, 50 patients with positive DT titres but without lymphadenopathy, and 100 patients with negative DTs. 
MATERIAL

Rabbit antihuman IgM, code no: 10-091, obtained from Dako, Copenhagen, Denmark.

Rabbit antitoxoplasma serum was prepared in conventionally raised rabbits which were initially DTnegative. They were injected intravenously with $10^{4}$ viable trophozoites of the RH strain of toxoplasma, obtained from mouse peritoneal fluid. After 10 days they were given another injection of $10^{5}$ trophozoites and after 28 days they were bled. The rabbit serum obtained had a DT titre of 1/32 000 . $\mathrm{F}\left(\mathrm{ab}^{\prime}\right)_{2}$ peroxidase conjugate: three separate batches were prepared from the rabbit sera by treatment with pepsin (Sigma, Poole Dorset) to prepare $F\left(a b^{\prime}\right)_{2}$ fragments ${ }^{9}$ which were then conjugated to horseradish peroxidase ${ }^{10}$ (grade RZ 3, EAB Ltd, Tafarnaubach Estate, Tredegar, Gwent).

The toxoplasma antigen was prepared from infected chorioallantoic membranes of hen's eggs by the method described for the preparation of complement fixation antigen. ${ }^{11}$

Phosphate-buffered saline (PBS), 0.01 M, pH 7.2. PBS containing $0.05 \%$ Tween 20 (PBST).

Normal rabbit serum (NRS), toxoplasma antibodyfree.

Bovine serum albumen (BSA).

Enzyme substrate consisted of 2,2'-azino-di-(3 ethyl benzthiazoline sulfonic acid) (ABTS) and was obtained from Sigma, Poole, Dorset. This substrate (20 mg) was dissolved in $100 \mathrm{ml}$ of $0.05 M$ citrate phosphate buffer, pH 4.0 , containing $0.02 \%$ hydrogen peroxide.

A PR 50 EIA automatic analyser and cuvettes, code no: 1414X9, obtained from Gilford Instruments Ltd, Twickenham, Middlesex.

\section{ACCA METHOD}

The Gilford PR 50 analyser provided a semiautomatic means of performing the ACCA in cuvettes and was used for the addition and aspiration and the final printout of the spectrophotometric reading. All incubation procedures were carried out at an ambient temperature of $20^{\circ} \mathrm{C}$ in a sealed, humid box. The results were expressed as a Test:Negative $(\mathrm{T}: \mathrm{N})$ ratio, where $\mathrm{N}$ was the mean absorbance of six IgM-negative control sera (three DT-negative and three DT-positive) tested at the same dilution as the test sera. A ratio of two or more was considered to be a positive result. All the sera were tested by ACCA on different occasions using three batches of $\mathrm{F}\left(\mathrm{ab}^{\prime}\right)_{2}$ conjugate.

The optimal dilution of reagents was determined by chessboard titrations using a weakly positive antitoxoplasma IgM serum at a dilution of $1 / 40$. The highest $T: N$ ratios were found when the antihuman IgM was used at $1 / 1000$, the toxoplasma antigen at
$1 / 40$ and the $F\left(a b^{\prime}\right)_{2}$ conjugate at $1 / 2000$ for two batches and $1 / 2500$ for the third.

Antihuman IgM was diluted in PBS and $200 \mu \mathrm{l}$ volumes added to cuvettes which were stored in a sealed, humid box for at least $24 \mathrm{~h}$ and then until used. The required number of cuvettes were washed nine times with PBST, and $200 \mu$ l volumes of PBST containing $1 \%$ NRS were added to the empty cuvettes. After three hours they were washed nine times as before, and $200 \mu \mathrm{l}$ volumes of test sera diluted in PBST containing 2\% NRS and 1\% BSA were added. Four hours later the cuvettes were again washed and $200 \mu \mathrm{l}$ volumes of antigen, diluted in PBST containing 5\% NRS and 5\% BSA were added.

After overnight incubation the cuvettes were washed and $200 \mu$ l volumes of $\mathrm{F}\left(\mathrm{ab}^{\prime}\right)_{2}$ conjugate, diluted in PBST containing 5\% NRS and 5\% BSA, were added, and incubated for one hour. The cuvettes were again washed and $250 \mu$ l volumes of freshly prepared enzyme-substrate were added. After incubation in a darkened box for $45 \mathrm{~min}$ the absorbances were read at $418 \mathrm{~nm}$ after zeroing the PR 50 with the substrate solution as a blank.

\section{DETERMINATION OF TOXOPLASMA ANTIBODY}

BY SDGC

Sucrose solutions were prepared in $0.15 \mathrm{M} \mathrm{NaCl}$ at concentrations (wt/vol) of $37.5 \%, 31 \%, 25 \%, 18 \%$ and $12.5 \%$. Density gradients were prepared by layering $700 \mu \mathrm{l}$ volumes of the sucrose solutions, in the stated order, into polypropylene tubes $(5.5 \mathrm{ml}$, MSE Scientific Instruments Ltd, Manor Royal, Crawley, Sussex). Serum $(200 \mu \mathrm{l})$ was mixed with an equal volume of $0.15 \mathrm{M} \mathrm{NaCl}$ and layered on top of the sucrose solutions. The tubes were centrifuged at $80000 \mathrm{~g}$ for $18 \mathrm{~h}$ in a Prepspin 50 centrifuge (MSE Scientific Instruments). Ten fractions of approximately $400 \mu$ l were collected by piercing the bottom of the tube and collecting drops. The first four fractions collected were tested in the presence and absence of $2-\mathrm{ME}$. Of each fraction, $150 \mu \mathrm{l}$ was added to two tubes, one tube received $50 \mu \mathrm{l}$ of 0.15 $M \mathrm{NaCl}$ and the other $50 \mu \mathrm{l}$ of $0.05 M 2-\mathrm{ME}$, the tubes were incubated at $37^{\circ} \mathrm{C}$ for one hour. The contents of both sets of tubes were examined by the DT, IgM-IFA and indirect haemagglutination test (IHA). When disruption of IgM occurred, reducing the titre obtained with the 2-ME fraction by eightfold or more, it was considered that specific antitoxoplasma IgM was present in the serum. The methods of performing the DT, IgM-IFA and IHA are given in PHLS Monograph series 13.11

\section{CHOICE OF SERUM DILUTION}

The SDGC method described is a sensitive means of 
identifying antitoxoplasma IgM and it was considered desirable for the ACCA to be at least as sensitive. A DT-negative control serum, together with five sera containing varying amounts of antitoxoplasma IgM, as determined by the SDGC method, were selected for titration in the ACCA. The T:N ratios for serum dilutions from $1 / 20$ to $1 / 1280$ of these six sera are shown in the Figure. Serum 1 had a DT titre of $1 / 256$ and a small amount of antitoxoplasma IgM which could only be detected at a low level by the DT following SDGC and was not detected by IHA or IgM-IFA. It had a T:N ratio of 2.4 at a dilution of $1 / 40$ and a $\mathrm{T}: \mathrm{N}$ ratio of 1.7 at $1 / 80$. A dilution of $1 / 40$ was chosen because it was the highest dilution at which this serum gave a T:N ratio above the cut-off value of 2 . Sera $2-5$ had DT titres of $1 / 1024$ or greater and contained increased

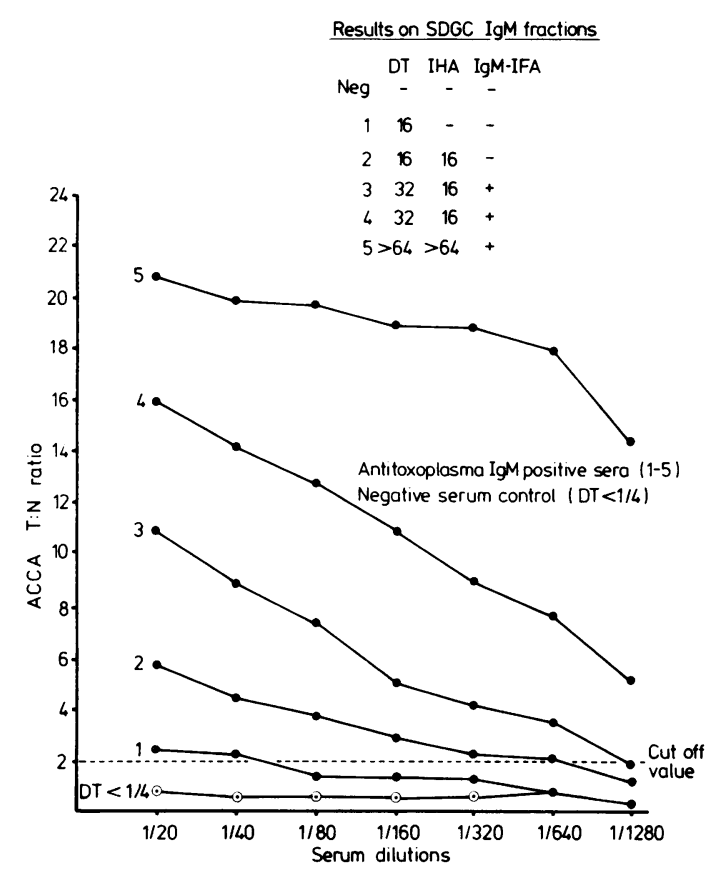

ACCA T:N ratios with 6 selected sera over a range of dilutions. amount of antitoxoplasma IgM. Serum 5, which had a very high level of antitoxoplasma IgM, gave absorbance readings approaching the upper limit on the scale of the spectrophotometer. The antitoxoplasma IgM level could not, therefore, be as accurately determined over the range $1 / 20$ to $1 / 640$ and the differences in the $T: N$ ratios obtained were not as great as with the other sera tested.

A serum dilution of $1 / 40$ proved to be a good choice because all the 78 sera in which antitoxoplasma IgM could be found by SDGC were identified by the ACCA when tested at this dilution (Table 1). This dilution can be conveniently made by adding $5 \mu \mathrm{l}$ of neat serum to the diluent in the cuvette.

\section{Results}

A total of 180 patients with suspected toxoplasmic lymphadenopathy were examined serologically for antitoxoplasma IgM (Table 1). Of 74 sera with a DT of 1/1024 or greater, 72 had an ACCA T:N ratio of $\geqslant 2.0$ and the presence of antitoxoplasma IgM was confirmed in 68 by positive antibody tests with the IgM serum fractions after SDGC. Sera from four patients gave positive results with ACCA and negative results with SGDC and sera from two patients gave negative results with both methods. The date of onset of lymphadenopathy could be established in four of these six patients and in each case it was more than a year previously. From a total of 82 ACCA positive sera, 10 had a DT of $\leqslant 1 / 256$. These sera were from patients with acute toxoplasmosis and subsequent specimens from them showed DT titres of $1 / 1024$ or greater in every case (Table 2). The ACCA and SDGC tests were positive with both specimens from all 10 patients whereas the IgMIFA on whole serum was positive with seven first specimens and only two second specimens from these patients.

The sensitivity and specificity of the ACCA was compared with the IgM-IFA on whole serum diluted $1 / 5$ and neat IgM fractions of sera after SDGC (Table 3). Of the sera from 180 patients with lym-

Table 1 Results of specimens from suspected cases of toxoplasmic lymphadenopathy

\begin{tabular}{|c|c|c|c|c|c|}
\hline DT titre & No tested & $A C C A>2.0$ & $A C C A<2.0$ & SDGC-positive & SDGC-negative \\
\hline $\begin{array}{l}1 / 4096 \\
1 / 1024 \\
1 / 256 \\
1 / 64 \\
1 / 16 \\
<1 / 4\end{array}$ & $\begin{array}{l}35 \\
39 \\
18 \\
18 \\
20 \\
50\end{array}$ & $\begin{array}{r}35 \\
37 \\
7 \\
3 \\
0 \\
0\end{array}$ & $\begin{array}{r}0 \\
2 \\
11 \\
15 \\
20 \\
50\end{array}$ & $\begin{array}{r}33 \\
35 \\
7 \\
3 \\
0 \\
\text { NT }\end{array}$ & $\begin{array}{r}2 \\
4 \\
11 \\
15 \\
20 \\
\text { NT }\end{array}$ \\
\hline Total & 180 & 82 & 98 & 78 & 52 \\
\hline
\end{tabular}

NT $=$ not tested. 
Table 2 Results of paired sera from acute cases of toxoplasmic lymphadenopathy

\begin{tabular}{|c|c|c|c|c|c|c|}
\hline Patient & Specimen & Interval (days) & DT titre & ACCA $T: N$ ratio & $S D G C$ & $\begin{array}{l}\text { IgM-IFA on } \\
\text { whole serum (1/5) }\end{array}$ \\
\hline \multirow[t]{2}{*}{1} & $1 \mathrm{st}$ & - & $1 / 256$ & $8 \cdot 0$ & + & + \\
\hline & 2nd & 8 & $1 / 2048$ & $8 \cdot 8$ & + & - \\
\hline \multirow[t]{2}{*}{2} & $1 \mathrm{st}$ & - & $1 / 256$ & $5 \cdot 1$ & + & \pm \\
\hline & 2nd & 14 & $1 / 2048$ & $6 \cdot 7$ & + & $\overline{-}$ \\
\hline \multirow[t]{2}{*}{3} & 1st & - & $1 / 64$ & 5.0 & + & + \\
\hline & 2nd & 15 & $1 / 2048$ & $6 \cdot 2$ & + & - \\
\hline \multirow[t]{2}{*}{4} & $1 \mathrm{st}$ & - & $1 / 256$ & $8 \cdot 2$ & + & \pm \\
\hline & 2nd & 27 & $1 / 1024$ & $7 \cdot 6$ & + & + \\
\hline \multirow[t]{2}{*}{5} & $1 \mathrm{st}$ & - & $1 / 256$ & $11 \cdot 2$ & + & + \\
\hline & 2nd & 18 & $1 / 2048$ & $10 \cdot \overline{3}$ & + & - \\
\hline \multirow[t]{2}{*}{6} & $1 \mathrm{st}$ & - & $1 / 64$ & 10.7 & + & + \\
\hline & 2nd & 14 & $1 / 1024$ & 11.4 & + & - \\
\hline \multirow[t]{2}{*}{7} & lst & - & $1 / 256$ & $7 \cdot 3$ & + & - \\
\hline & 2nd & 21 & $1 / 2048$ & 6.0 & + & - \\
\hline \multirow[t]{2}{*}{8} & lst & - & $1 / 256$ & $5 \cdot 0$ & + & - \\
\hline & 2nd & 22 & $1 / 8096$ & $3 \cdot 5$ & + & - \\
\hline \multirow[t]{2}{*}{9} & $1 \mathrm{st}$ & - & $1 / 64$ & 3.9 & + & + \\
\hline & 2nd & 19 & $1 / 2048$ & $6 \cdot 0$ & + & + \\
\hline \multirow[t]{2}{*}{10} & $1 \mathrm{st}$ & - & $1 / 256$ & 9.7 & + & - \\
\hline & 2nd & 116 & $1 / 2048$ & $7 \cdot 8$ & + & - \\
\hline
\end{tabular}

Table 3 Results of IgM-IFA on whole serum and SDGC fractions

\begin{tabular}{lcccc}
\hline Specimens & No tested & $\begin{array}{c}\text { Positive IgM-IFA } \\
\text { on whole serum }\end{array}$ & $\begin{array}{c}\text { Positive IgM-IFA } \\
\text { on SDGC fractions }\end{array}$ & $\begin{array}{c}\text { ACCA }>2 \cdot 0 \\
\text { Lymphadenopathies }\end{array}$ \\
RF sera & 180 & 34 & 54 & 82 \\
ANF sera & 20 & 10 & 10 & 0 \\
DT-positive sera & 3 & 2 & 2 & 0 \\
DT-negative sera & 50 & $1 *$ & NT & 0 \\
\hline
\end{tabular}

NT $=$ not tested.

*This serum was subsequently found to contain RF.

phadenopathy only 34 were positive when the whole serum was tested by IgM-IFA, 54 were positive when competitive IgG had been removed by SDGC. The IgM-IFA therefore, proved to be far less sensitive than the ACCA. False-positive results occurred with 10 of 20 sera containing RF and two of three sera containing ANF when tested by IgM-IFA whereas all were negative when tested by ACCA. Sera from 100 patients without lymphadenopathy and with negative DTs and 50 patients without lymphadenopathy and DT titres ranging from $1 / 16$ to $1 / 256$ were also tested. All were negative with ACCA, one gave a positive result with IgM-IFA but this specimen was subsequently shown to contain RF.

\section{Discussion}

The use of a $F\left(a b^{\prime}\right)_{2}$ peroxidase conjugate has undoubtedly increased the specificity of ACCA for the detection of antitoxoplasma IgM and has eliminated the problems caused by RF and ANF. Other authors using a similar method, originally found the IgG fraction of antitoxoplasma rabbit serum, conjugated to alkaline phosphatase, did not cause falsepositive results with sera containing RF or ANF. ${ }^{12}$
They have since reported false-positive results with this method but have also shown that these could be avoided by using $F\left(a b^{\prime}\right)_{2}$ conjugate. ${ }^{13}$ In a recent letter the same authors report that when $F\left(a^{\prime}\right)_{2}$ conjugate was used, false-positive results could occur with sera from patients who had received rabbit antithymocyte globulin. ${ }^{14}$ These patients were heart transplant recipients and had developed IgM antirabbit antibodies.

The sensitivity of the ACCA is increased by the addition of NRS and BSA to the diluents. This results in a reduction of the mean absorbance of the negative sera whereas the absorbances of positive sera remain unaffected. Higher $T: N$ ratios and increased sensitivity are therefore attained. Acute cases of toxoplasmic lymphadenopathy can be correctly diagnosed early in the disease by the examination of only a single specimen of serum. This diagnosis cannot be made on the DT result of the first specimen alone and a second specimen is required to establish a rise in titre. An IgM-IFA on whole serum can identify some early cases but it is of lower sensitivity and associated with false-positive and falsenegative results.

Studies of the duration of antitoxoplasma IgM after onset of lymphadenopathy are in progress and 
will be published in due course. Preliminary findings suggest that the antitoxoplasma IgM response varies considerably in individual patients. In one case it was detectable for only three months, in another it was detectable for one year after onset. In the majority of patients so far studied, when sequential specimens were tested, antitoxoplasma IgM could be detected by both ACCA and SDGC methods for about six months after onset. However, the ACCA method appears to detect antitoxoplasma IgM for a slightly longer period than the SDGC method.

The antigen for the tests was prepared from toxoplasma-infected membranes of hens' eggs. This antigen was used because it was readily available. However, most of the sera were also tested using an antigen prepared from water lysates of toxoplasma derived from mouse peritoneal exudates and similar results were obtained. The source of the antigen is probably not significant but antigen derived from tissue culture has not yet been tried.

Large scale investigations can be carried out by ACCA with the aid of the PR 50 automatic analyser. It requires very little attention and is able to process a large number of sera. Only $5 \mu \mathrm{l}$ of serum is required for each test and batches of up to 1000 sera can be initiated on one day and read on the following day. The cuvettes have been shown to give more reliable and consistent results than microtitre plates. ${ }^{1516}$ However, very occasionally, we did encounter an unexpected high reading, particularly in either the first or last cuvette in a strip of ten. For this reason tests on all sera giving high readings were repeated twice.

The results clearly demonstrate that the ACCA as described, is sensitive, specific, and reproducible. It is more sensitive and specific than the IgM-IFA, less laborious than the SDGC method and applicable to large scale screening.

We are indebted to Dr W Kwantes, Director of the Swansea Public Health Laboratory for helpful criticism and advice, and to Louise Davies for manuscript typing.

\section{References}

' Remington JS, Miller MJ, Brownlee I. IgM antibodies in acute toxoplasmosis. II Prevalence and significance in acquired cases. J Clin Med 1968;71:855-66.

${ }^{2}$ Camargo ME, Jesen PGG, Rocca A. Rheumatoid factors as a cause for false-positive IgM antiToxoplasma fluorescent tests. A technique for specific results. Rev Inst Med Trop Sao Paulo 1972:14:310-3.

${ }^{3}$ Araujo FG, Barnett EV, Gentry LO, Remington JS. Falsepositive antiToxoplasma fluorescent antibody tests in patients with antinuclear antibodies. Appl Microbiol 1971;22:270-5.

${ }^{4}$ Cohen IR. Nouins LC. Julian AJ. Competition between, and effectiveness of IgG and IgM antibodies in indirect fluorescent antibody and other tests. J Immunol 1967;98: 143-9.

${ }^{5}$ Gupta JD, Peterson V, Stout M, Murphy AM. Single sample diagnosis of recent rubella by fractionation of antibody on Sephadex G-200 columns. J Clin Pathol 1971;24:547-50.

- Caul EO, Hobbs SJ, Roberts PC, Clarke SKR. Evaluation of a simplified sucrose gradient method for the detection of rubella specific IgM in routine diagnostic practice. J Med Virol 1978;2:153-63.

' Duermeyer W, van der Veen J. Specific detection of IgMantibodies by ELISA, applied in hepatitis A. Lancet 1978;ii:684.

${ }^{8}$ Sabin A, Feldman HA. Dyes as microchemical indicators of a new immunity phenomenon affecting a protozoan parasite (Toxoplasma). Science 1948;108:660-3.

${ }^{9}$ Rossi G, Choi TK, Nisonoff A. Crystals of fragment F (ab'): preparation from pepsin digests of human myeloma proteins. Nature 1969;223:837-8.

${ }^{10}$ Wilson MB, Nakane PK. Recent developments in the peroxidase method of conjugating horseradish peroxidase to antibodies. In: Knapp W, ed. Immunofluorescence and related techniques Amsterdam: Elsevier, 1978:215-24.

"Fleck DG, Kwantes W. The laboratory, diagnosis of Toxoplasmosis Public Health Laboratory Service Monograph series 1980:13.

${ }^{12}$ Naot Y, Remington JS. An Enzyme Linked Immunosorbent Assay for detection of IgM antibodies to Toxoplasma gondii. Use for diagnosis of acute acquired Toxoplasmosis. J Infect Dis 1980;142:757-66.

${ }^{13}$ Naot Y, Bennet EV, Remington JS. Method of avoiding false positive results occurring in Immunoglobulin $M$ Enzyme Linked Immunosorbent Assays due to the presence of both rheumatoid factor and antinuclear antibodies. J Clin Microbiol 1981;14:73-8.

${ }^{14}$ Naot Y, Luft BJ, Remington JS. False-positive serological tests in heart transplant recipients. Lancet 1981;ii:590-1.

${ }^{15}$ Francis J. Letter to the editor. Med Lab Sci 1981;37:303.

${ }^{16}$ Woodhouse CS, Ford CHJ, Newman CE. ELISA solid phase precision. Med Lab Sci 1981;38:147.

Requests for reprints to: Mr RA Payne, Public Health Laboratory, Cockett Road, Swansea SA2 OFA, Wales. 